\title{
An Application of Chain Code-Based Local Descriptor and Its Extension to Face Recognition
}

\author{
Paweł Karczmarek $^{\mathrm{a}, 1}$, Adam Kiersztyn ${ }^{\mathrm{a}}$, Witold Pedrycz ${ }^{\mathrm{b}, \mathrm{c}, \mathrm{d}}$, and Michał Dolecki ${ }^{\mathrm{a}}$ \\ ${ }^{a}$ The John Paul II Catholic University of Lublin, Institute of Mathematics and Computer \\ Science, ul. Konstantynów 1H, 20-708, Lublin, Poland \\ ${ }^{b}$ Department of Electrical \& Computer Engineering, University of Alberta, Edmonton T6R \\ 2V4 AB, Canada \\ ${ }^{c}$ Department of Electrical and Computer Engineering, Faculty of Engineering, King \\ Abdulaziz University, Jeddah, 21589, Saudi Arabia \\ ${ }^{d}$ Systems Research Institute, Polish Academy of Sciences, Warsaw, Poland
}

\begin{abstract}
Local descriptors are widely used technique of feature extraction to obtain information about both local and global properties of an object. Here, we discuss an application of the Chain Code-Based Local Descriptor to face recognition by focusing on various datasets and considering different variants of this description method. We augment the generic form of the descriptor by adding a possibility of grouping pixels into blocks, i.e., effectively describing larger neighborhoods. The results of experiments show the efficiency of the approach. We demonstratethat the obtained results are comparable or even better than those delivered by other important algorithms in the class of methods based on the Bag-ofVisual-Words paradigm.
\end{abstract}

Keywords: face recognition; local descriptor; chain code; chain code-based local descriptor.

\section{Introduction}

Face recognition has been one of the most important problems of biometrics in the recent decades fully recognized by wide community of researchers. Its vast plethora of applications covers, among others, forensic sciences, driver's licenses and passport verification, missing identification, surveillance systems, social networks, etc. A relative easiness of usage (noninvasiveness), and low technological costs imply the immense development of various facial recognition approaches. However, many of them still suffer from some of typical problems occurring in image recognition such as lighting conditions, quality of old images, aging, pose of the subject, face occlusion, computing overhead, etc. There are many trends in face recognition. The most significant and comprehensively examined are the approaches originated from geometrical methods [1], principal component analysis (eigenfaces[2]), linear discriminant analysis (Fisherfaces[3]), EBGM (elastic bunch graph matching [4]), SVMs (support vector machines [5]), Gabor wavelets [6,7], information aggregation and fusion [8], neural networks [9], sparse representation [10], deep learning [11,12], Granular Computing and linguistic descriptors $[13,14]$, and local descriptors $[15,16]$.For instance, the latter class of

\footnotetext{
${ }^{1}$ Corresponding author. Tel.: +48 8144545 08; fax: +48 814454551 .

E-mail addresses: pawelk@kul.pl (P. Karczmarek), adam.kiersztyn@kul.pl (A. Kiersztyn), wpedrycz@ualberta.ca (W. Pedrycz), michal.dolecki@kul.pl (M. Dolecki)
} 
methods is,in general, invariant to lighting and pose changes. Other advantages of local descriptors are their relatively low computational cost and very intuitive processing scheme taking as an input the neighborhood of a given pixel and resulting in the value being the description of this surrounding. Next, histograms of such descriptionsin the local areas are formed. Finally, the histograms are concatenated producing a description of the whole image.

The origin of the idea of local descriptors could be encountered in the area oftexture analysis $[17,18]$, and, in the sequel they could be found in applications to face recognition. A milestone was a publication authored by Ahonen et al.[15]. This paper describes a local descriptor, which converts the value of the grayscale level of the analyzed pixel to the value dependent on its neighborhood. The analysis of the neighborhood, see Fig. 1, determines which pixels from the surroundings have values greater or smaller than the examined, central pixel. Based on this finding, the pixels constituting the neighborhood are coded using zeros and ones. This new coding allows us to create the new value describing the tested pixel as digit value of the byte built from the eight bits ( 0 or 1 values).

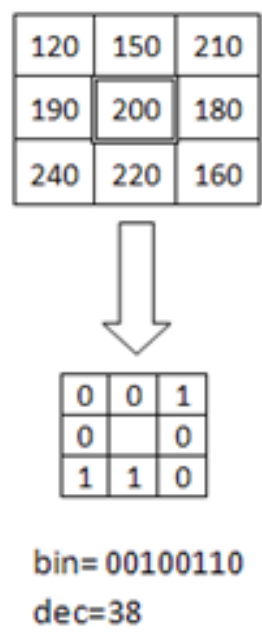

Fig. 1 Visualization of the concept of the LBP

On the basis of LBP, a lot of variations of the approach were established. The most important that are worth mentioning include central symmetric LBP [16,19], improved local binary pattern (ILBP) [20], multi-scale block LBP [21,22], or Weber local descriptor (WLD) [23]. In addition, many papers describing linkages of local descriptors with different filters, like Gabor wavelets, have been published [6,24,25,26,27].The extensive research on application of local descriptors in pattern recognition is still ongoing, as evidenced by numerous new studies in this field, e.g., [28,29,30,31,32,33,34,35,36,37,38,39,40,41,42].Particularly, noteworthy is the recent trend of combining deep learning with local descriptors, see, for instance,[43,44,45]. Comprehensive survey study can be found in $[7,46]$.

An interesting approach to local descriptors was describedin [47]. In this method, called Full Ranking (FR), the use of the classical histogram is replaced by using a dictionary of available words, which is a direct incorporation of the Bag-of-Visual-Words (BoVW) paradigm [48]. As in the LBP case, the pixel neighborhoodsare examined (Fig. 2). However, the pixels are 
sorted and the indexes constitute a "word". Finally, the word is replaced by the closest one appearing in the dictionary. The histograms of the words form the inputs to the classifiers.

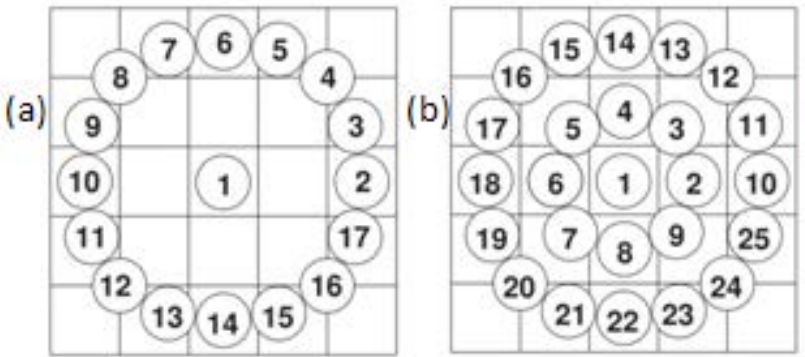

\begin{tabular}{|c|c|c|c|c|}
\hline 8 & 7 & 6 & 5 & 4 \\
\hline 9 & & & & 3 \\
\hline 10 & & 1 & & 2 \\
\hline 11 & & & & 17 \\
\hline 12 & 13 & 14 & 15 & 16 \\
\hline
\end{tabular}

(d) \begin{tabular}{|l|l|l|}
\hline 7 & 8 & 9 \\
\hline 6 & 1 & 2 \\
\hline 5 & 4 & 3 \\
\hline
\end{tabular}

Fig. 2 Neighborhoods considered in the Chan et al. [47] algorithm. After sorting by increasing values of the pixels, the indices form the word, the closest word from the available dictionary replaces the value of the analyzed pixel. Neighborhoods of the forms (a-c) are proposed in [47] while the neighborhood (d) has been drawn from the classic LBP algorithm. Hereafter, the forms of neighborhoods are denoted as $A, B, C$, and $D$, respectively.

Finally, in [49] proposed was a so-called Chain Code-Based Local Descriptor (CCBLD). This novel method applies the "words" built from the chain codes starting from the analyzed pixels. The code is created taking into account, for instance, the consecutive minima or maxima of the pixel values. It implies the replacement of the integer (usually $0-255$ ) values by words created by only four letters. This type of description of the neighborhood of any pixel should help to obtain local description of an image. However, longer codes contain information on the more global structures than the neighborhood of an arbitrary shape, for instance 3x3. The details of the method are discussed in the next section.

The main objective of this study is to develop a novel extension of basic Chain Code-Based Local Descriptor (CCBLD), which is realized by an application of blocks of pixels instead of the single ones. The originality of the approach lays in the calculation of the chain-codes describing the neighborhoods of pixels taking into account the blocks of pixels. This simple procedure results in the significant increase of the accuracies for the most of the considered datasets. Moreover, this study shows the application of the BoVW paradigm to the more complicated structures such as apparently non-related blocks of pixels by building "words" describing the relations laying behind them. Furthermore, we present a comprehensive analysis of the dependency of the recognition rate on the dictionary length and the division of the image onto the subregions for which separate histograms are created and then concatenated into the holistic description of the image. Our aim is also to demonstrate the usefulness and applicability of CCBLD to various environments, lighting conditions, or age by testing its accuracy on numerous well-known face databases such as AT\&T, FERET, Yale, CAS-PEAL, ColorFERET, Essex, and FG-NET.

Some preliminary experimental studies reported in [49] for the AT\&T dataset and demonstrating some potential, are significantly expanded in this paper. Here, we discuss more comprehensive tests showing the accuracy of the CCBLD-based face recognition. Finally, we investigate some design aspects by studying an influence of preprocessing (e.g., cropping) on the recognition with CCBLD. 
The paper is organized as follows. In Section 2, we discuss the main properties of Chain Code-Based Local Descriptor and present its extensions. The experimental results are described in Section 4. Conclusions and directions of future work are covered in Section 5.

\section{Chain Code-Based Local Descriptor and its extension}

Let us briefly recall the main idea behind the CCBLD. It is worth noting here that this local descriptor, in contrary to others, is created not on the basis of the size of the region itself (e.g., rectangle or circle) but the description of a selected pixel may (at least theoretically) come from a larger number of pixels (or blocks) depending upon the local properties of an image. In more detail, assume that we start from any arbitrary pixel $I(x, y)$ and that in one of the pixels $I_{L}(x-1, y), I_{U}(x, y-1), I_{R}(x+1, y)$, or $I_{D}(x, y+1)$ there is a higher gray-scale value than the $I(x, y)$ (and, it is the highest value in this cross-neighborhood). Then, the first letter of the description of the pixel $I(x, y)$ corresponds to one of the four considered pixels, namely "L", "U”, "R", or " $D$ ". Next, the process continues starting from the pixel of this maximal value. Of course, the procedure has its limits since the gray-scale range of values and the number of pixels in any image are finite. Moreover, it is easy to see that the cycles are not possible. However, in case of two or more maxima, one can set some priorities as to the choice of the highest value. Finally, when the starting pixel exhibits the highest value, the description results in an empty chain. Similarly, any pixel of an image can be described by taking into account the consecutive minima using the same letters. The process is illustrated in Fig. 3.

\begin{tabular}{|l|l|l|l|l|}
\hline 52 & 43 & 46 & 52 & 59 \\
\hline 51 & $\mathbf{4 0}$ & 46 & $\mathbf{5 6}$ & 42 \\
\hline 48 & $\mathbf{4 3}$ & $\mathbf{5 0}$ & $\mathbf{5 3}$ & 44 \\
\hline 45 & $\mathbf{4 6}$ & $\mathbf{4 9}$ & 49 & 49 \\
\hline 46 & 48 & 47 & 50 & 42 \\
\hline
\end{tabular}

Max path: RURU

Min path: UU

Fig. 3 The process of finding the consecutive minima and maxima from the starting point (pixel of value 46)

Since the number of such string descriptions can be huge, a soundalternative is to introduce a dictionary of chosen possible words. The words not appearing in this dictionary can be matched to the nearest one present in the dictionary. The process of string comparison can be realized following a well-known Levenshtein distance (edit distance). Let us recall that in the determination of this distance we take into account the minimal number of insertions, replacements, and deletions which are needed to make to words equal [50,51].

A final description of an image comes as a concatenation of histograms of dictionary words appearing in subregions of the image. It helps preserve both local (at the level of subregions) and global (as a result of concatenation) properties of an image. Formally, we write it down as

$$
D=\left[w_{1,1}^{+}, \ldots, w_{1, \frac{n}{2}}^{+}, w_{1,1}^{-}, \ldots, w_{1, \frac{n}{2}}^{-}, \ldots, w_{i, j}^{+}, \ldots, w_{i, j}^{-}, \ldots, w_{r, 1}^{+}, \ldots, w_{r, \frac{n}{2}}^{+}, w_{r, 1}^{-}, \ldots, w_{r, \frac{n}{2}}^{-}\right]
$$


where $i=1, \ldots, r$ is a number of image subregion, $j=1, \ldots, n / 2$ is a number of word in a dataset. The sign + denotes a word created by using the consecutive maxima rule while the sign stands for the word built with the rule of minima. Similarly to what has been presented in [47], we create the dictionary by random picking up images and pixels in the database. As the classifier we propose is the nearest neighbor with the Hellinger similarity measure (also known as Bhattacharyya coefficient) comparison of the histograms (regarded as two vectors $\left.=\left(x_{1}, \ldots, x_{n}\right), \boldsymbol{y}=\left(y_{1}, \ldots, y_{n}\right) \in \mathbb{R}^{n}\right)$ is completed as follows [52]

$$
d(\boldsymbol{x}, \boldsymbol{y})=\sqrt{1-\frac{\sum_{i=1}^{n} \sqrt{x_{i} y_{i}}}{\sqrt{\sum_{i=1}^{n} x_{i} \sum_{i=1}^{n} y_{i}}}}
$$

As it will be shown in the experiments section, a simple modification to the above procedure can play a pivotal role in increasing the recognition rates. Instead of considering single pixels, we can take the blocks of pixels, which are then represented as the arithmetic means of the blocks of size $p \times p$, i.e.,

$$
\bar{I}(x, y)=\frac{I(x-p, y-p)+\cdots+I(x, y)+\cdots+I(x+p, y+p)}{(2 p+1)^{2}},
$$

for instance,

$$
\bar{I}_{L}(x, y)=\frac{I(x-3 p-1, y-p)+\cdots+I(x-p-1, y)+\cdots+I(x-p-1, y+p)}{(2 p+1)^{2}},
$$

etc. An example of a process of word construction corresponding to a certain pixel is depicted inFig. 4. The overall classification process is presented in Fig. 5. 


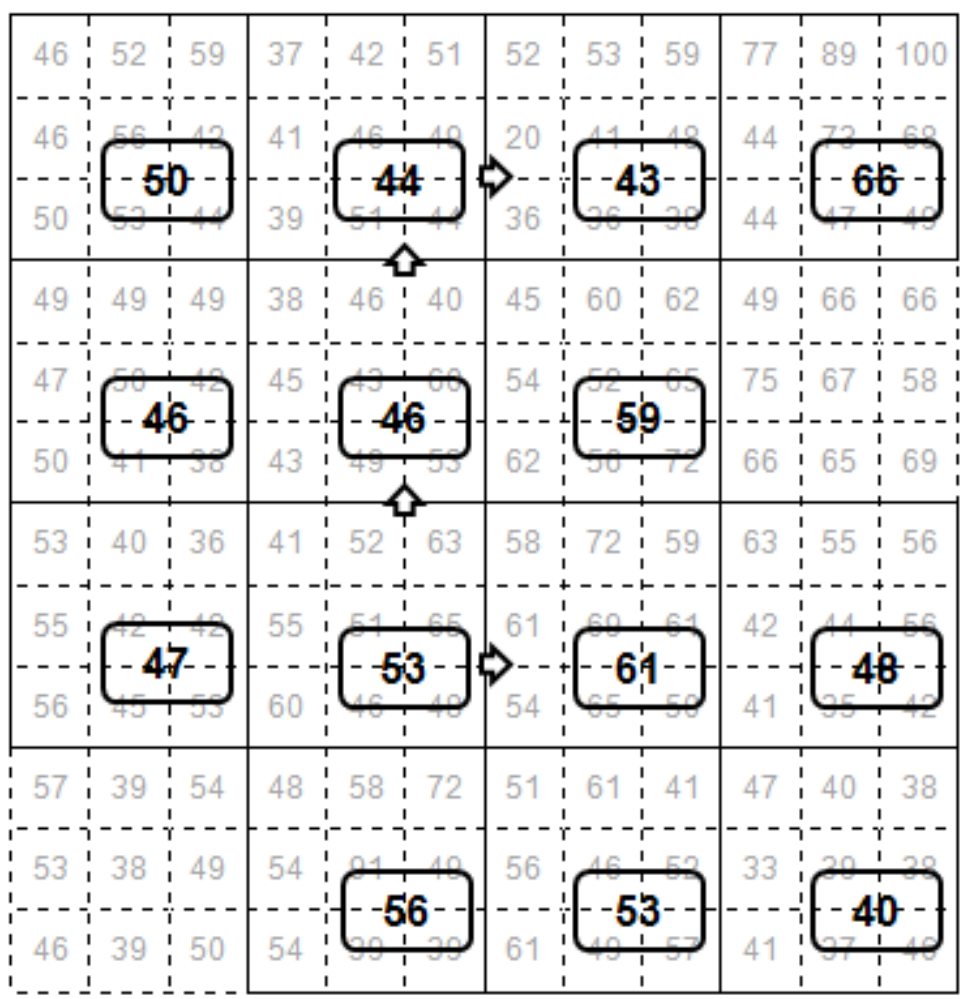

Max path: $\mathrm{R}$

Min path: UUR

Fig. 4Example of finding the consecutive minima and maxima of averages of blocks, here the size of a block is $p=3$
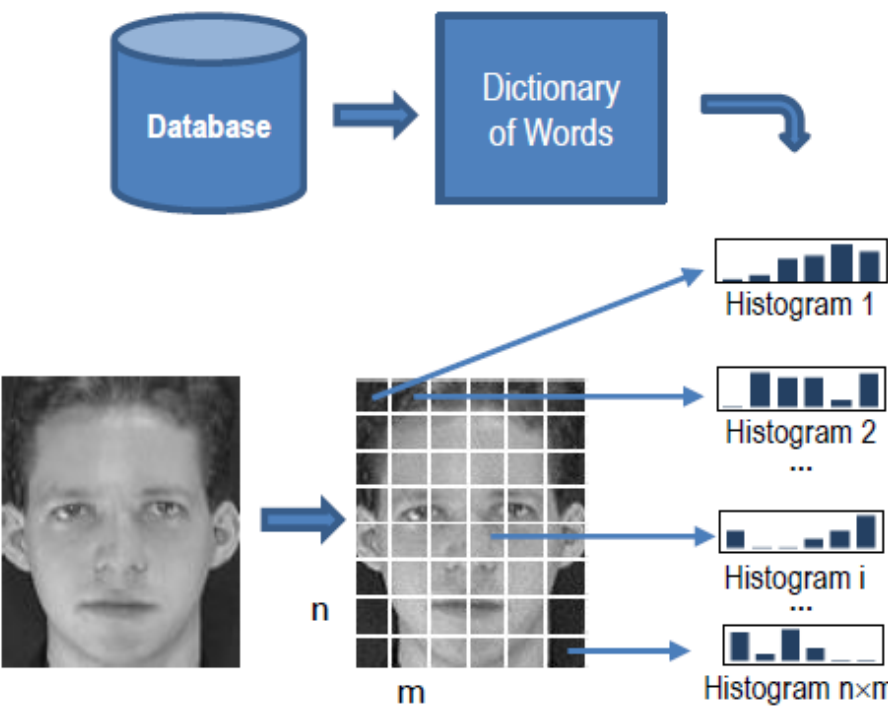

Histogram $\mathrm{n} \times \mathrm{m}$

Fig. 5An overall processing scheme

\section{Experimental studies}

To demonstrate the efficiency of the proposed approach, we present experimental results obtained for seven commonly used face datasets. The results are compared with those obtained for the LBP method [15] and with Full Ranking descriptor presented in [47].

\subsection{AT\&T database}


AT\&T [53] is one of the most exploited facial datasets in scientific publications. It consists of 400 images of 40 people with 10 images per subject. The images are taken with relatively different mimic, emotion expression, pose, and light. They are 92 pixels in width and 112 pixels in height. For the purpose of our experiments we have divided the dataset choosing randomly 5 images of each person to the gallery and the rest 5 images to the testing set. The experiment was repeated 20 times. In each series of test we have created randomly a new dictionary of words. The experiments were done for various lengths of dictionary and divisions of the images in the subregions. However, no matter of the relation of the width to the height of the images, we have always selected the division in the form $n \times n$, i.e., the subregions have the same proportions as the original whole images.Table 1, Table 2, Table 3, and Table 4 contain the rank 1 recognition rates obtained with CCBLD local descriptor having the size of block surrounding the starting pixel of value $p=1, p=2, p=3$, and $p=4$, respectively, and for the dictionaries ranging from 10 to 300 words. Some additional results for these descriptors with longer dictionaries and split of the images are given in Table 5, where only the best results are detailed. We specified them by analyzing the progress of the recognition rates values for the dictionaries of lower length. In case of relatively simple dataset which is AT\&T the dictionary of length 100 for basic CCBLD and $400-600$ for block version of the descriptor is a well choice. The best division for this dataset is $3 \times 3$ or $4 \times 4$ subregions of the equal size. Fig. 6visualizes the comparison for the first 10 ranks of 5 versions of CCBLD, LBP,and FR local descriptors. Hereafter the notion CCBLD(Npx,MxN,K) refers to the descriptor with $N$-pixel wide neighborhood of central (starting) point, division of the image on $\mathrm{M}$ rows and $\mathrm{N}$ columns, and dictionary of length $K$. The plot shows the potential applicability of our CCBLD method pointing that it is a sound alternative for one of the best descriptors published ever.

The last series of tests with the AT\&T data presented here concerns the verification of the methods on the images being preprocessed, namely, the faces are cropped (90 x $94 \mathrm{px}$ ), scaled, and the histogram are equalized. Our method produces over $98 \%$ recognition rate and for all the ranks outperforms the other descriptors. Fig. 7stresses that when the images are preprocessed, a good level of recognition (over 98\%) is achieved.

Table 1Relation betweenrank 1 recognition rates and the dictionary length for the CCBLD with block size $p=1$

\begin{tabular}{|c|c|c|c|c|c|c|c|c|c|c|c|c|c|c|}
\hline & Dictio & ary leng & & & & & & & & & & & & \\
\hline Division & 10 & 20 & 30 & 40 & 50 & 60 & 70 & 80 & 90 & 100 & 150 & 200 & 250 & 300 \\
\hline $1 \times 1$ & 52.95 & 69.33 & 74.78 & 77.58 & 79.68 & 81.45 & 82.28 & 82.80 & 82.93 & 83.75 & 85.23 & 84.60 & 85.50 & 85.53 \\
\hline $2 \times 2$ & 83.00 & 88.80 & 89.70 & 90.63 & 91.00 & 91.50 & 91.45 & 92.00 & 92.63 & 92.28 & 92.93 & 92.75 & 92.80 & 93.18 \\
\hline $3 \times 3$ & 90.23 & 92.58 & 93.38 & 94.15 & 94.28 & 94.65 & 94.85 & 94.75 & 95.43 & 95.23 & 95.68 & 95.75 & 95.93 & 95.83 \\
\hline $4 \times 4$ & 92.95 & 94.70 & 95.48 & 95.68 & 95.83 & 95.83 & 95.98 & 96.03 & 96.10 & 96.20 & 95.93 & 96.15 & 96.00 & 95.60 \\
\hline $5 \times 5$ & 93.68 & 95.10 & 95.33 & 95.30 & 95.53 & 95.60 & 95.55 & 95.55 & 95.53 & 95.58 & 95.45 & 95.15 & 95.10 & 94.85 \\
\hline $6 \times 6$ & 93.03 & 94.15 & 94.80 & 95.30 & 95.18 & 95.03 & 95.25 & 95.05 & 95.35 & 95.33 & 95.18 & 94.73 & 94.53 & 93.98 \\
\hline $7 \times 7$ & 93.55 & 94.30 & 94.20 & 94.53 & 94.28 & 94.48 & 94.18 & 94.28 & 94.15 & 94.28 & 93.93 & 93.75 & 93.53 & 93.10 \\
\hline $8 x 8$ & 92.58 & 93.35 & 93.50 & 93.60 & 93.73 & 93.43 & 93.73 & 93.53 & 93.55 & 93.45 & 93.28 & 92.78 & 92.80 & 92.53 \\
\hline $9 \times 9$ & 92.45 & 93.18 & 93.38 & 93.40 & 93.20 & 93.40 & 93.28 & 93.20 & 93.13 & 93.25 & 92.85 & 92.63 & 92.43 & 92.25 \\
\hline $10 \times 10$ & 92.38 & 92.70 & 92.78 & 92.80 & 92.80 & 92.50 & 92.63 & 92.68 & 92.50 & 92.63 & 92.08 & 91.83 & 91.68 & 91.03 \\
\hline
\end{tabular}


Table 2Relation between rank 1 recognition rates and the dictionary length for the CCBLD with block size $p=3$

\begin{tabular}{|c|c|c|c|c|c|c|c|c|c|c|c|c|c|c|}
\hline \multirow[b]{2}{*}{ Division } & \multicolumn{14}{|c|}{ Dictionary length } \\
\hline & 10 & 20 & 30 & 40 & 50 & 60 & 70 & 80 & 90 & 100 & 150 & 200 & 250 & 300 \\
\hline $1 \times 1$ & 72.13 & 85.28 & 88.93 & 91.08 & 92.03 & 92.88 & 93.38 & 93.13 & 94.08 & 94.13 & 94.93 & 95.30 & 95.35 & 95.75 \\
\hline $2 \times 2$ & 92.20 & 94.80 & 96.43 & 96.73 & 96.60 & 96.95 & 97.25 & 97.10 & 97.13 & 97.43 & 97.60 & 97.93 & 98.00 & 97.93 \\
\hline $3 \times 3$ & 96.05 & 97.28 & 97.60 & 97.88 & 97.83 & 97.93 & 98.00 & 97.85 & 97.85 & 97.95 & 97.95 & 98.20 & 98.15 & 98.20 \\
\hline $4 \times 4$ & 96.70 & 97.63 & 97.75 & 98.03 & 98.10 & 98.23 & 98.18 & 98.20 & 98.08 & 98.18 & 98.28 & 98.18 & 98.20 & 98.25 \\
\hline $5 \times 5$ & 96.58 & 97.10 & 97.20 & 97.05 & 97.35 & 97.28 & 97.08 & 97.28 & 97.43 & 97.23 & 97.20 & 97.00 & 97.23 & 97.20 \\
\hline $6 \times 6$ & 96.28 & 96.83 & 96.90 & 96.83 & 96.70 & 96.88 & 96.63 & 96.83 & 96.88 & 96.93 & 96.90 & 96.93 & 96.68 & 96.83 \\
\hline $7 \times 7$ & 96.50 & 96.58 & 96.43 & 96.58 & 96.50 & 96.58 & 96.48 & 96.60 & 96.53 & 96.43 & 96.40 & 96.55 & 96.40 & 96.45 \\
\hline $8 x 8$ & 95.25 & 96.03 & 96.00 & 95.90 & 96.00 & 96.00 & 95.88 & 96.03 & 95.90 & 95.98 & 95.98 & 96.00 & 95.93 & 95.93 \\
\hline $9 \times 9$ & 95.38 & 95.50 & 95.80 & 95.70 & 95.53 & 95.65 & 95.70 & 95.55 & 95.60 & 95.55 & 95.68 & 95.73 & 95.70 & 95.55 \\
\hline $10 \times 10$ & 95.45 & 95.65 & 95.35 & 95.53 & 95.50 & 95.60 & 95.38 & 95.35 & 95.45 & 95.43 & 95.35 & 95.35 & 95.48 & 95.30 \\
\hline
\end{tabular}

Table 3Relation between rank 1 recognition rates and the dictionary length for the CCBLD with block size $p=5$

\begin{tabular}{|c|c|c|c|c|c|c|c|c|c|c|c|c|c|c|}
\hline & Dictio & ry leng & & & & & & & & & & & & \\
\hline Division & 10 & 20 & 30 & 40 & 50 & 60 & 70 & 80 & 90 & 100 & 150 & 200 & 250 & 300 \\
\hline $1 \mathrm{x} 1$ & 72.90 & 85.98 & 89.28 & 91.18 & 91.73 & 92.65 & 92.88 & 93.25 & 93.43 & 93.78 & 94.78 & 95.03 & 95.75 & 95.65 \\
\hline $3 \times 3$ & 95.78 & 97.38 & 97.30 & 97.43 & 97.68 & 97.88 & 97.83 & 97.75 & 97.85 & 98.10 & 98.00 & 98.00 & 98.00 & 98.00 \\
\hline $4 \times 4$ & 96.90 & 97.35 & 97.48 & 97.30 & 97.50 & 97.53 & 97.43 & 97.55 & 97.45 & 97.50 & 97.50 & 97.53 & 97.50 & 97.45 \\
\hline $5 \times 5$ & 95.90 & 96.25 & 96.33 & 96.45 & 96.48 & 96.45 & 96.60 & 96.70 & 96.53 & 96.58 & 96.73 & 96.73 & 96.73 & 96.73 \\
\hline $7 \times 7$ & 95.33 & 96.03 & 95.95 & 96.13 & 95.93 & 95.95 & 96.00 & 96.00 & 95.93 & 95.95 & 96.10 & 95.93 & 95.90 & 95.93 \\
\hline $8 x 8$ & 95.23 & 95.63 & 95.65 & 95.55 & 95.63 & 95.65 & 95.58 & 95.58 & 95.48 & 95.55 & 95.50 & 95.35 & 95.38 & 95.63 \\
\hline $9 \times 9$ & 95.13 & 95.33 & 95.50 & 95.53 & 95.30 & 95.48 & 95.35 & 95.30 & 95.25 & 95.28 & 95.15 & 95.18 & 95.28 & 95.08 \\
\hline $10 \times 10$ & 94.73 & 95.18 & 95.05 & 95.05 & 94.98 & 95.20 & 94.93 & 94.95 & 94.68 & 95.00 & 94.88 & 94.78 & 94.63 & 94.70 \\
\hline
\end{tabular}

Table 4Relation between rank 1 recognition rates and the dictionary length for the CCBLD with block size $p=7$

\begin{tabular}{|c|c|c|c|c|c|c|c|c|c|c|c|c|c|c|}
\hline \multirow[b]{2}{*}{ Division } & \multicolumn{14}{|c|}{ Dictionary length } \\
\hline & 10 & 20 & 30 & 40 & 50 & 60 & 70 & 80 & 90 & 100 & 150 & 200 & 250 & 300 \\
\hline $1 \times 1$ & 70.13 & 84.13 & 87.08 & 90.10 & 91.50 & 91.85 & 92.78 & 92.55 & 92.78 & 93.28 & 94.03 & 94.68 & 95.18 & 95.28 \\
\hline $2 \times 2$ & 92.18 & 94.65 & 95.88 & 96.45 & 96.58 & 96.78 & 96.85 & 97.15 & 97.20 & 97.05 & 97.48 & 97.65 & 97.53 & 97.70 \\
\hline $3 \times 3$ & 96.33 & 97.35 & 97.73 & 97.93 & 98.00 & 98.15 & 98.13 & 98.10 & 98.33 & 98.33 & 98.33 & 98.33 & 98.33 & 98.28 \\
\hline $4 \times 4$ & 96.65 & 97.70 & 97.80 & 97.73 & 97.83 & 97.85 & 97.68 & 98.05 & 97.88 & 98.05 & 97.88 & 97.90 & 97.88 & 97.95 \\
\hline $5 \times 5$ & 96.18 & 96.85 & 96.80 & 97.03 & 96.95 & 97.23 & 97.03 & 97.23 & 97.18 & 97.20 & 97.18 & 97.15 & 97.05 & 96.95 \\
\hline $6 \times 6$ & 96.60 & 96.68 & 96.75 & 96.88 & 96.60 & 96.65 & 96.65 & 96.70 & 96.68 & 96.58 & 96.58 & 96.33 & 96.38 & 96.50 \\
\hline $7 \times 7$ & 95.80 & 96.13 & 96.10 & 96.38 & 96.30 & 96.35 & 96.25 & 96.55 & 96.28 & 96.35 & 96.33 & 96.28 & 96.43 & 96.40 \\
\hline $8 \times 8$ & 95.65 & 95.88 & 95.85 & 96.13 & 96.13 & 96.15 & 96.28 & 96.30 & 95.98 & 96.05 & 96.18 & 96.18 & 96.23 & 96.33 \\
\hline $9 \times 9$ & 95.60 & 95.83 & 95.70 & 95.70 & 95.90 & 95.78 & 95.85 & 95.48 & 95.65 & 95.65 & 95.73 & 95.60 & 95.70 & 95.63 \\
\hline $10 \times 10$ & 95.08 & 95.55 & 95.75 & 95.63 & 95.58 & 95.50 & 95.58 & 95.40 & 95.45 & 95.58 & 95.35 & 95.55 & 95.40 & 95.40 \\
\hline
\end{tabular}

Table 5 Additional rank 1 recognition rates for selected division of images and sizes of descriptor's block for AT\&T database

\begin{tabular}{lllllllll}
\hline \multicolumn{7}{c}{ Dictionary length } \\
\hline Block size $(p)$ & Division & 400 & 500 & 600 & 700 & 800 & 900 & 1000 \\
\hline 3 & $2 \times 2$ & 97.95 & 98.2 & 98.15 & 98.23 & 98.08 & 98 & 98.08 \\
3 & $3 \times 3$ & 98.13 & 97.98 & - & - & - & - & - \\
3 & $4 \times 4$ & 98.28 & 98.15 & 98.15 & 98.23 & - & - & - \\
5 & $2 \times 2$ & 98 & 97.93 & - & - & - & - & - \\
5 & $3 \times 3$ & 98.15 & 98.05 & 98.23 & 98.15 & - & - & - \\
5 & $4 \times 4$ & 97.6 & 97.45 & - & - & - & - & -
\end{tabular}




\begin{tabular}{lllllllll}
7 & $2 \times 2$ & 97.68 & 97.5 & - & - & - & - & - \\
7 & $3 \times 3$ & $\mathbf{9 8 . 4 5}$ & 98.3 & 98.23 & 98.3 & 98.23 & 98.23 & 98.23 \\
7 & $4 \times 4$ & 97.85 & 97.95 & 97.75 & 97.78 & - & - & - \\
9 & $2 \times 2$ & - & 96.73 & - & - & - & - & - \\
9 & $3 \times 3$ & 98.35 & 98.38 & 98.4 & 98.4 & 98.28 & 98.28 & 98.28 \\
9 & $4 \times 4$ & - & 97.83 & - & - & - & - & - \\
11 & $2 \times 2$ & - & 96.15 & - & - & - & - & - \\
11 & $3 \times 3$ & - & 97.73 & - & - & - & - & - \\
11 & $4 \times 4$ & - & 97.53 & - & - & - & - & - \\
\hline
\end{tabular}

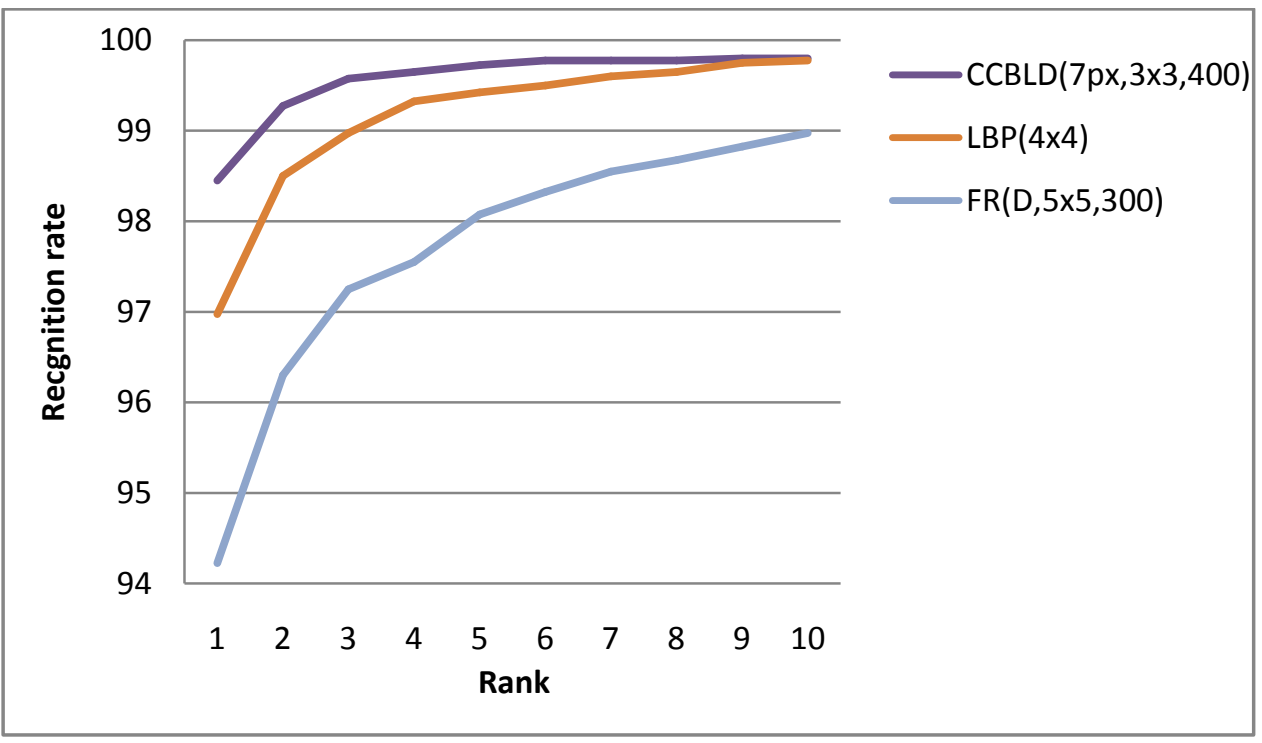

Fig. 6 Rank 1 - rank 10 recognition rates (\%) obtained for chosen local descriptors for AT\&T database

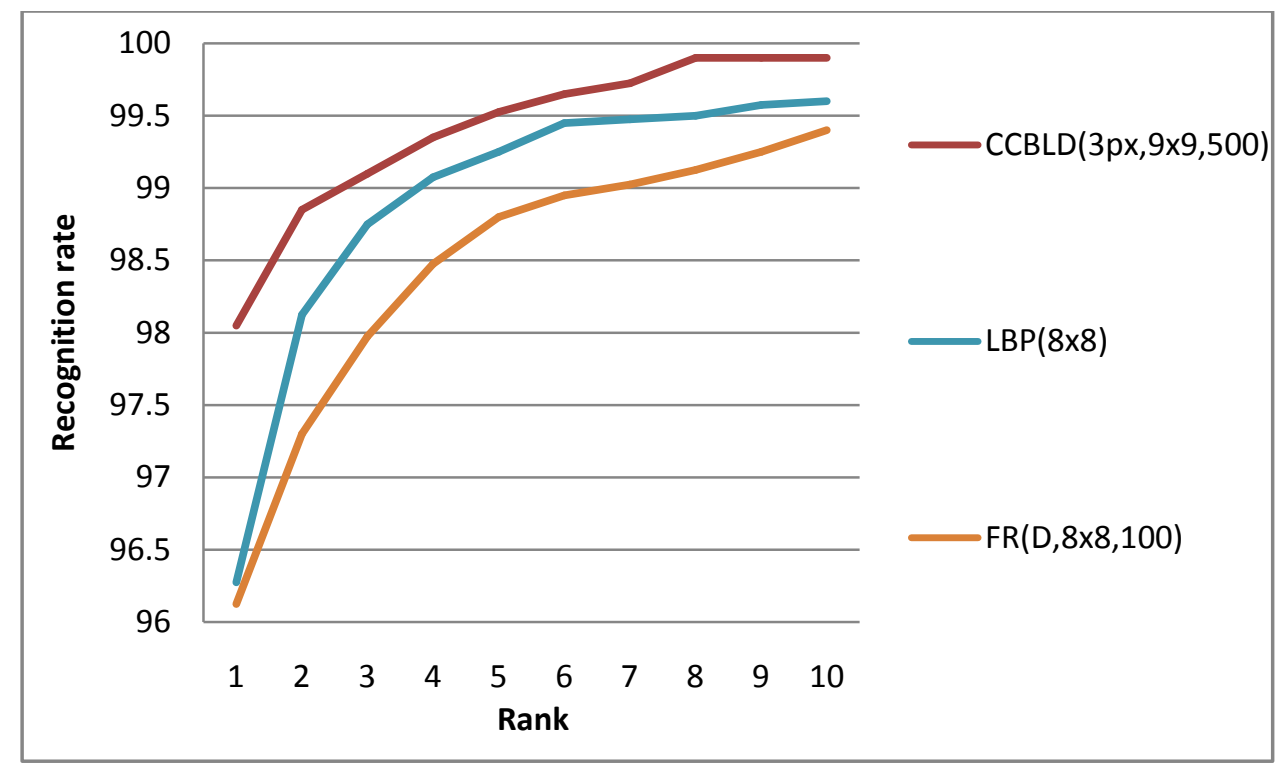

Fig. 7 Rank 1 - rank 10 recognition rates (\%) obtained for chosen local descriptors for preprocessed images from AT\&T dataset

\subsection{FERET database}


Now, we consider various subsets of the well-known Facial Recognition Technology (FERET) Database [54,55]. The first part of experiments was carried for merged grayscale image subsets called $b a, b k$, and $b j$, containing 600 photographs of 200 people (3 per subject). Similarly as in the AT\&T case, we have run 20 series of experiments choosing randomly 2 images of each person for the training and 1 image for the testing set, respectively. The results show that the CCBLD in its simplest form, namely 1 pixel size neighborhood, outperforms the rest of the methods. Of course, the recognition rates are not high (73.75\% for rank 1), but they show the potential hidden in this approach and suggest its ability to connection with other image processing/recognition methods to increase its efficiency (see Fig. 8). Similar experiments were conducted for the same set of images after a preprocessing step has been completed. First, the eyes were detected, next the images were rescaled, and the faces were cropped (size 100x140 px), finally the histograms of the images were equalized. The results are presented at Fig. 9. They show, again, that the applicability of our approach and the preprocessing step can slightly improve the efficiency of the method by yielding over $75 \%$ recognition rate.

The next series of experiments was run on the ColorFERET dataset. We have chosen 2,722 frontal photographs of 994 people (from 2 to 22 images per subject in various distancesfrom the camera). The only preprocessing was grayscale conversion of all images. The results are depicted in Fig. 10. Finally, we have run similar tests for cropped and scaled (to the size $100 x 140$ px) faces from the grayscale FERET pictures set called $f a, f b, b a, b k$, and $b j$ (the operation was done for 3880 images). The results of comparison of CCBLD and other methods under consideration are presented inFig. 11. All gathered results illustrate an evident potential of the proposed method.

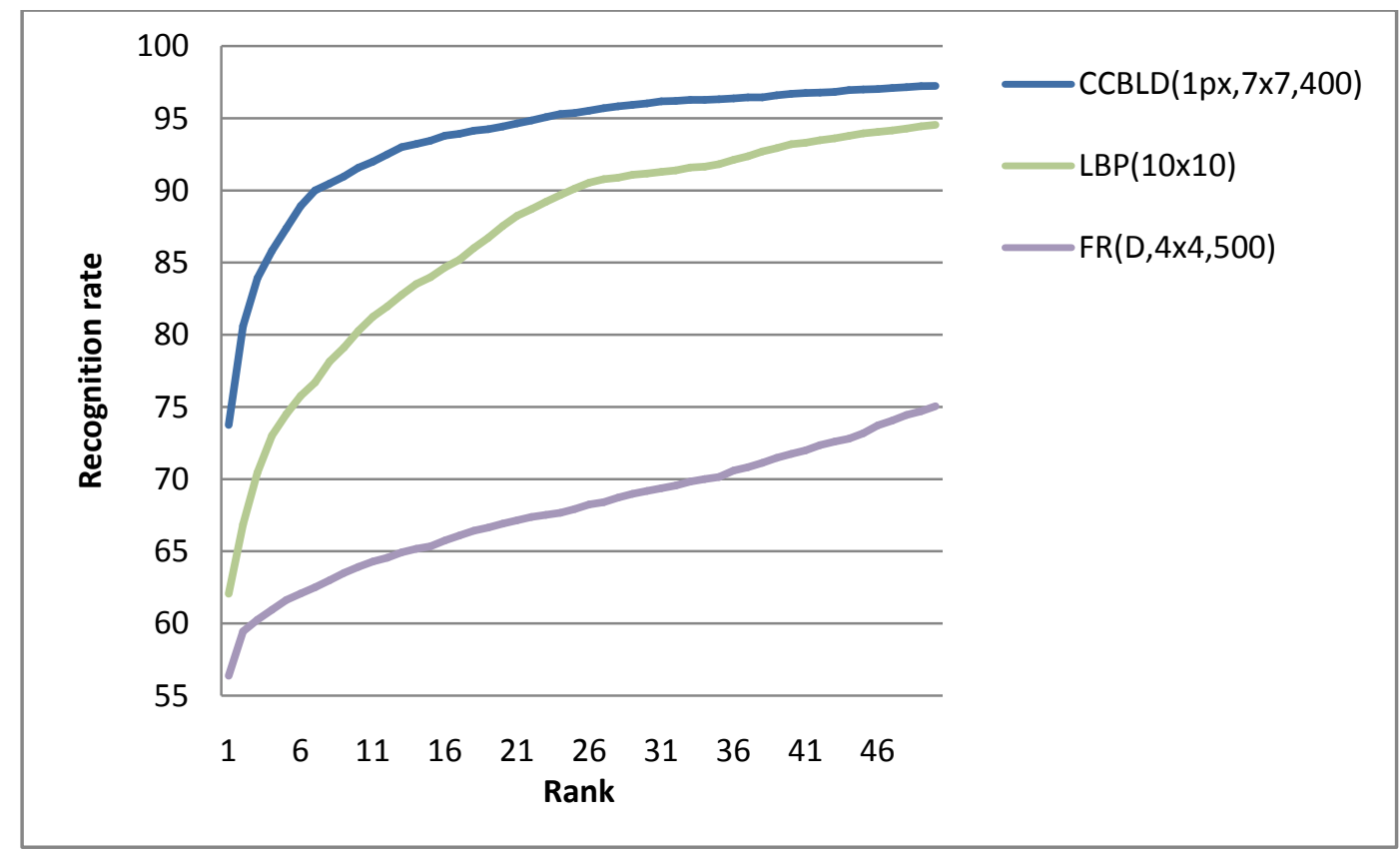

Fig. 8 Rank 1 - rank 50 cumulated recognition rates (\%) obtained for chosen local descriptors for preprocessed images from grayscale FERET subset 


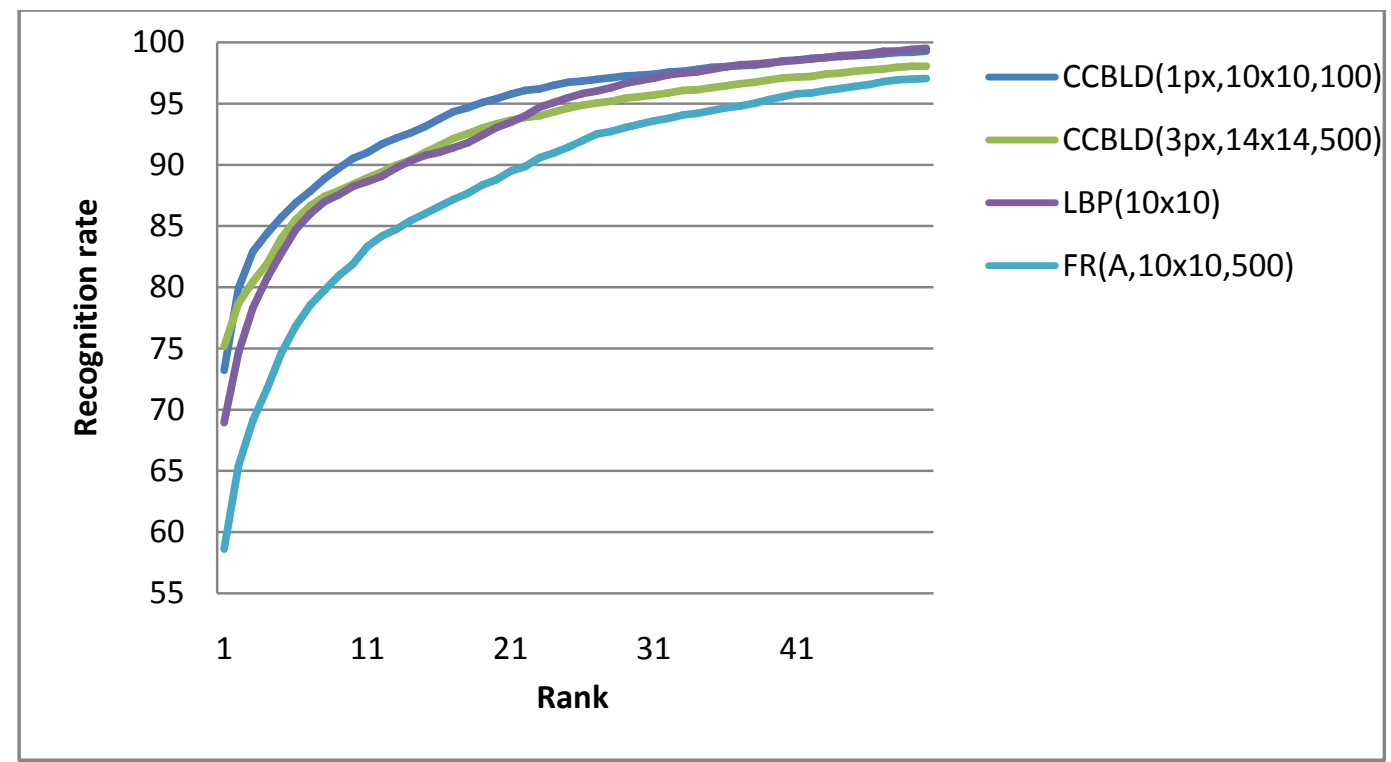

Fig. 9 Rank 1 - rank 50 recognition rates (\%) obtained for chosen local descriptors for preprocessed images from grayscale FERET subset containing 600 images after preprocessing

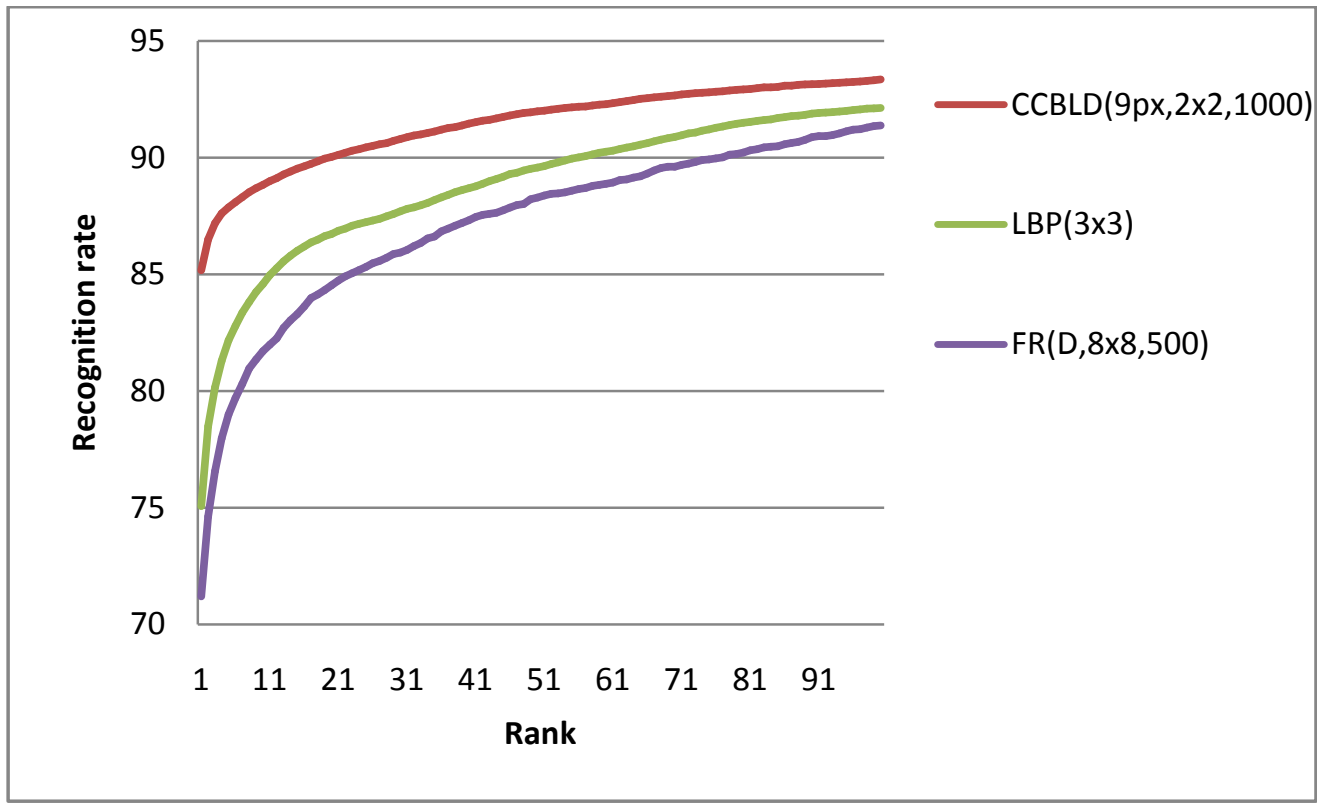

Fig. 10 A comparison of rank 1 - rank 100 recognition rates (\%) for the chosen methods in group of CCBLD, LBP, and FR for ColorFERET dataset 


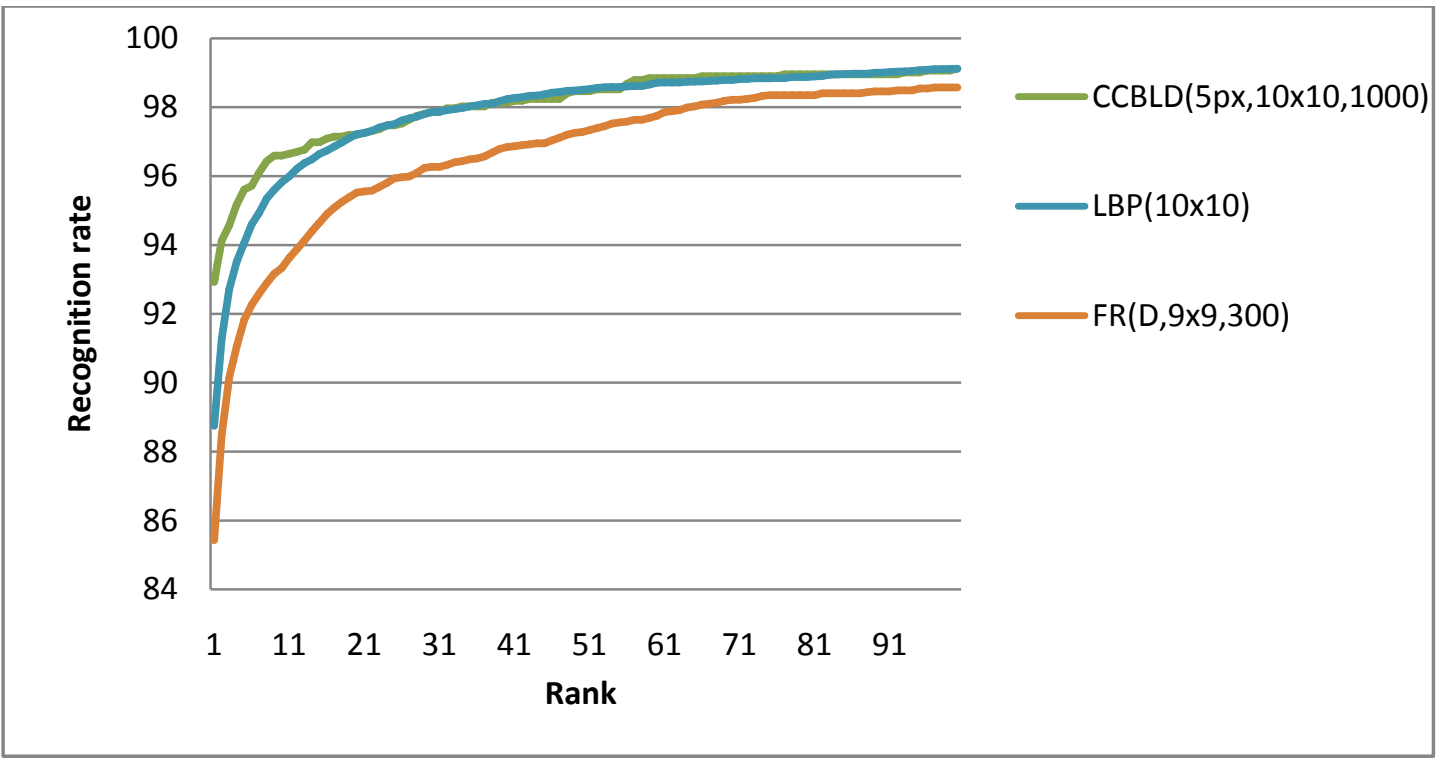

Fig. 11 A comparison of rank 1 - rank 100 recognition rates for the chosen methods in group of CCBLD, LBP, and FR for grayscale FERET dataset

\subsection{Yale Face Database}

Here we discuss the recognition problem using the 165 images of 15 people from the Yale Face Database [3]. The recognition rates obtained with chosen feature extractors are shown in Fig. 12. Here, we observe the dependency of the recognition rate on the division of the image onto the sub-blocks and, which is although very important, large dependency on the length of the dictionary.

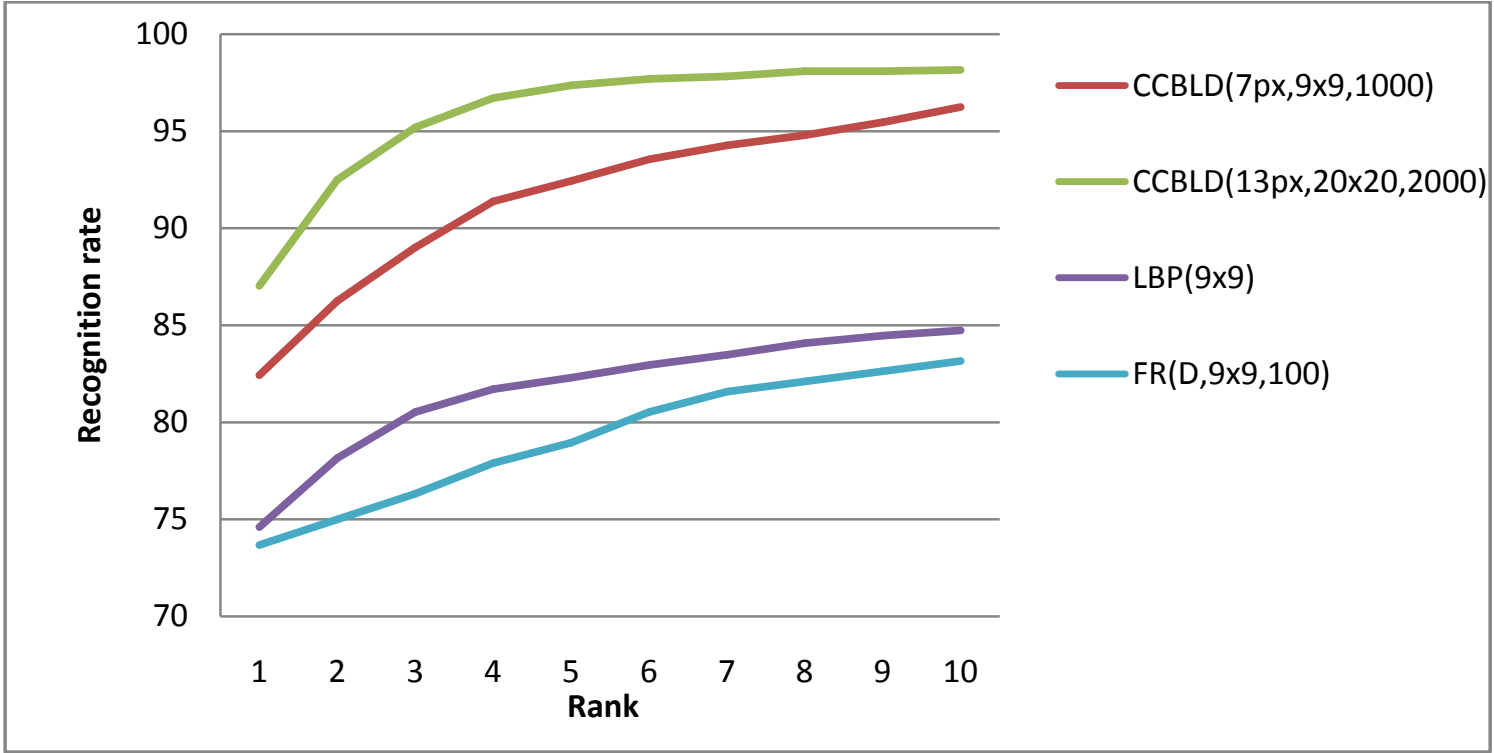

Fig. 12 A comparison of rank 1 - rank 10 cumulatedrecognition rates (\%) for the chosen methods in group of CCBLD, LBP, and FR for Yale Face Database

\subsection{CAS-PEAL database}


Here we report the results obtained for the CAS-PEAL database, see [56], precisely its subset containing 9,029 images of 1,040 people (from 3 to 49 per subject). The results are given in Fig. 13. For lower rank one can note that the chain-code based on blocks of pixels gives better results. However, one pixel-based chain code yields similar results for higher than 20 ranks.

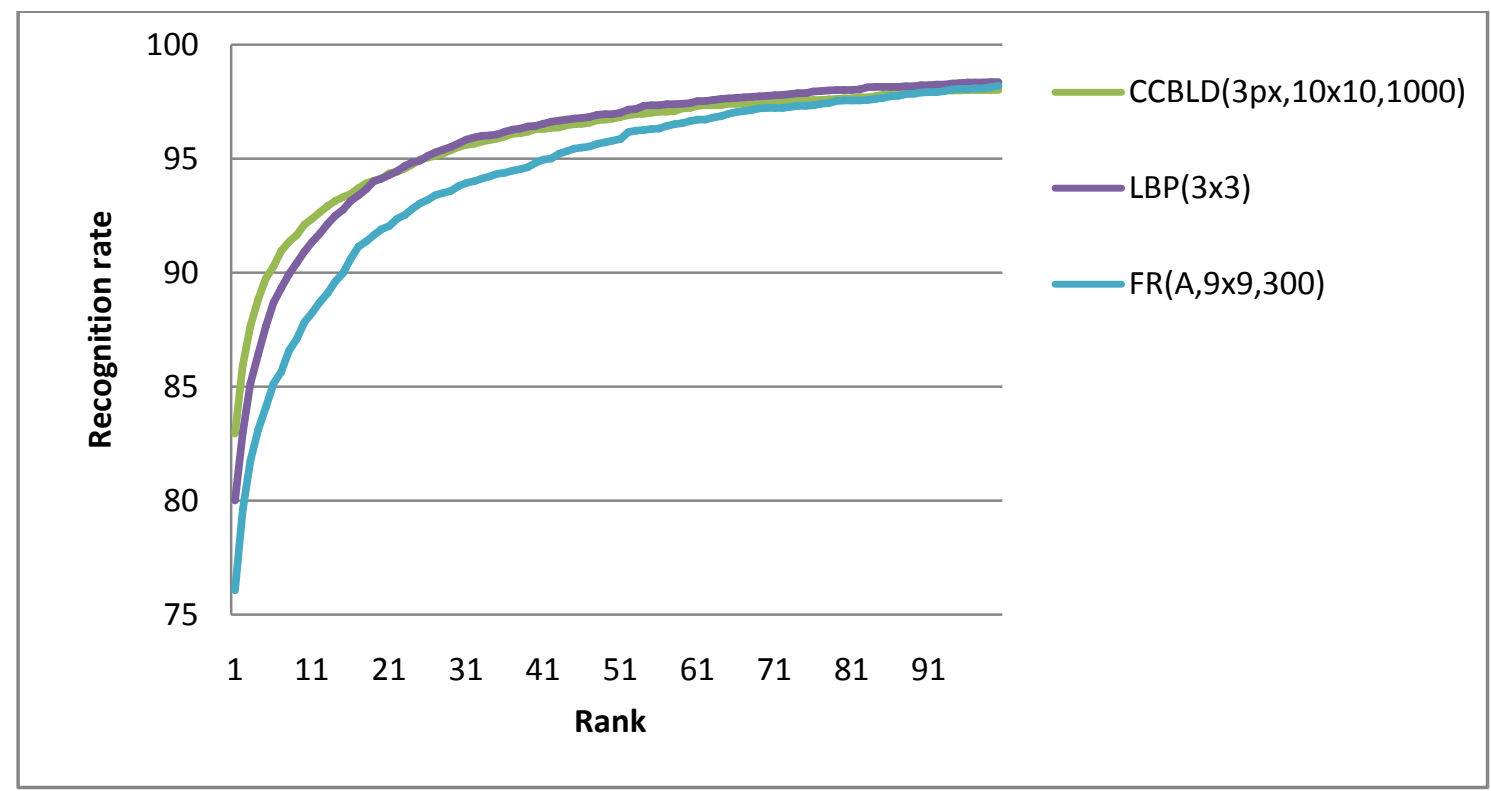

Fig. 13 A comparison of recognition rates (\%) for the chosen methods in group of CCBLD, LBP, and FR obtained with CAS-PEAL dataset

\subsection{Essex Collection of Facial Images}

The results deal with the subset faces94 from the Essex Collection of Facial Images[57] of 153 people (20 images per person) of size 180x200 px. They are depicted in Fig. 14.The plot shows that all the considered local descriptors bring good results here. However, also in this case the CCBLD outperforms both Full Ranking and LBP. 


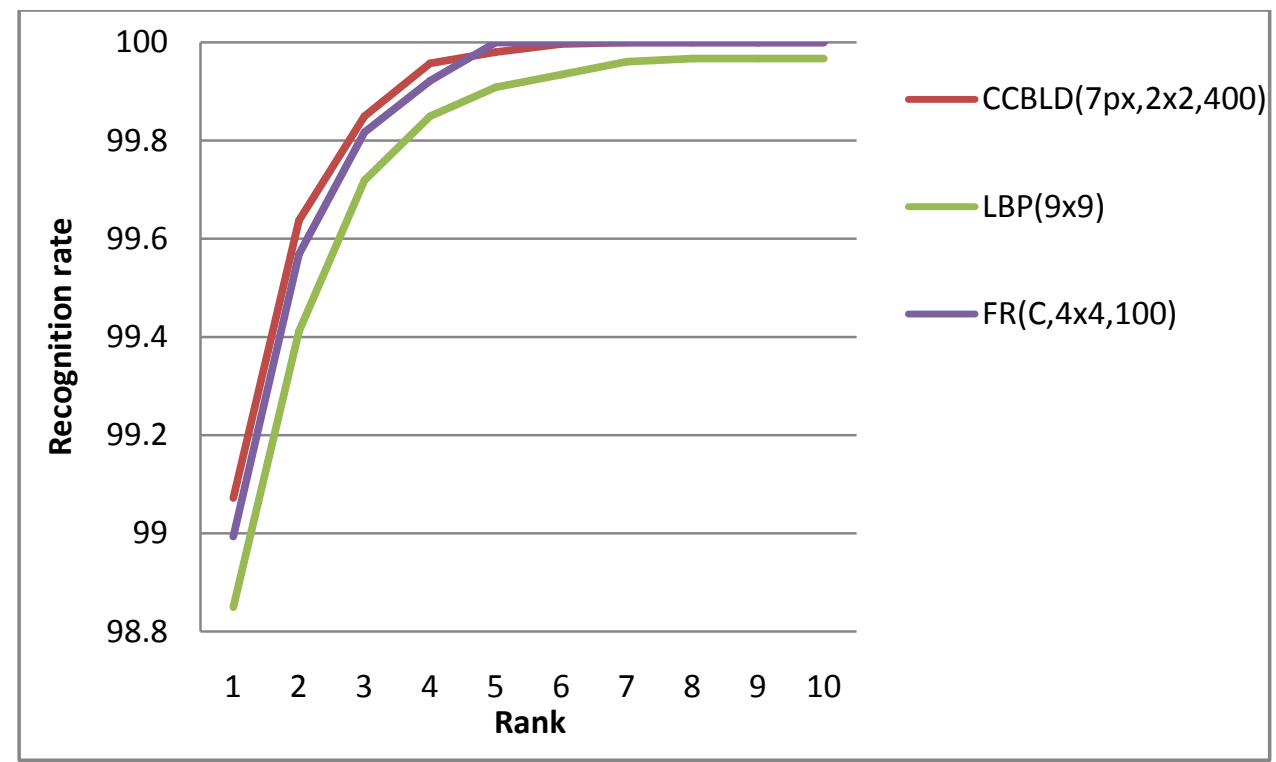

Fig. 14 A comparison of rank 1 - rank 10 recognition rates (\%) for the chosen methods in group of CCBLD, LBP, and FR obtained with Essex Collection of Facial Images

\subsection{Face and Gesture Aging Database (FG-NET)}

The FG-NET database [58] consists of 1,002 images of subjects at the age between 0 and 69 with 6 to 18 images per person. The maximum time between photos being taken is 54 years. The results are presented in Fig. 15. In the second series of the experiments, the photographs were converted to the grayscale, cropped to the size $128 \times 160 \mathrm{px}$, scaled, and the histograms were equalized. The recognition rates are illustrated in Fig. 16. Despite the fact that local descriptors themselves are not sufficient to bear the aging problem they can be potentially very useful in combination with other methods (see, for instance, [7]). The results show that CCBLD can be a good choice in comparison to other local descriptors, particularly when the images are preprocessed. 


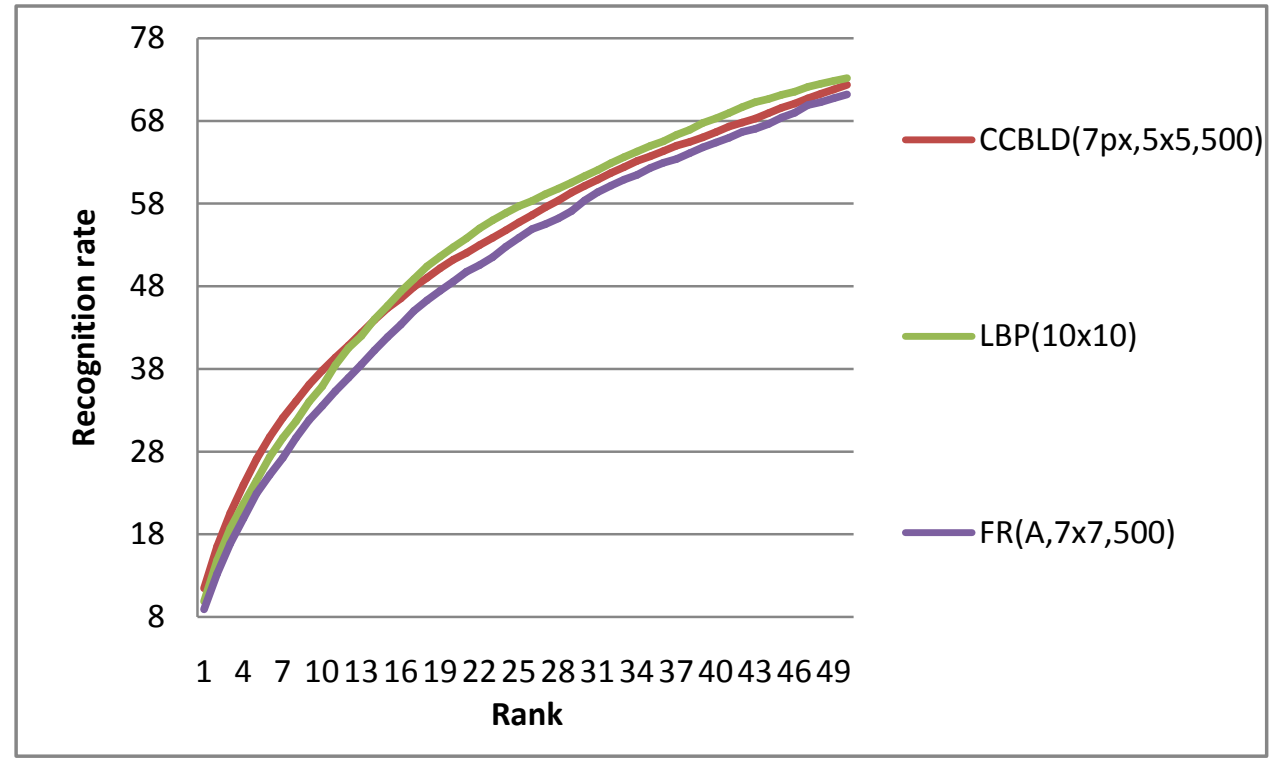

Fig. 15 A comparison of rank 1 - rank 50 recognition rates (\%) for the chosen methods in group of CCBLD, LBP, and FR obtained with FG-NET dataset

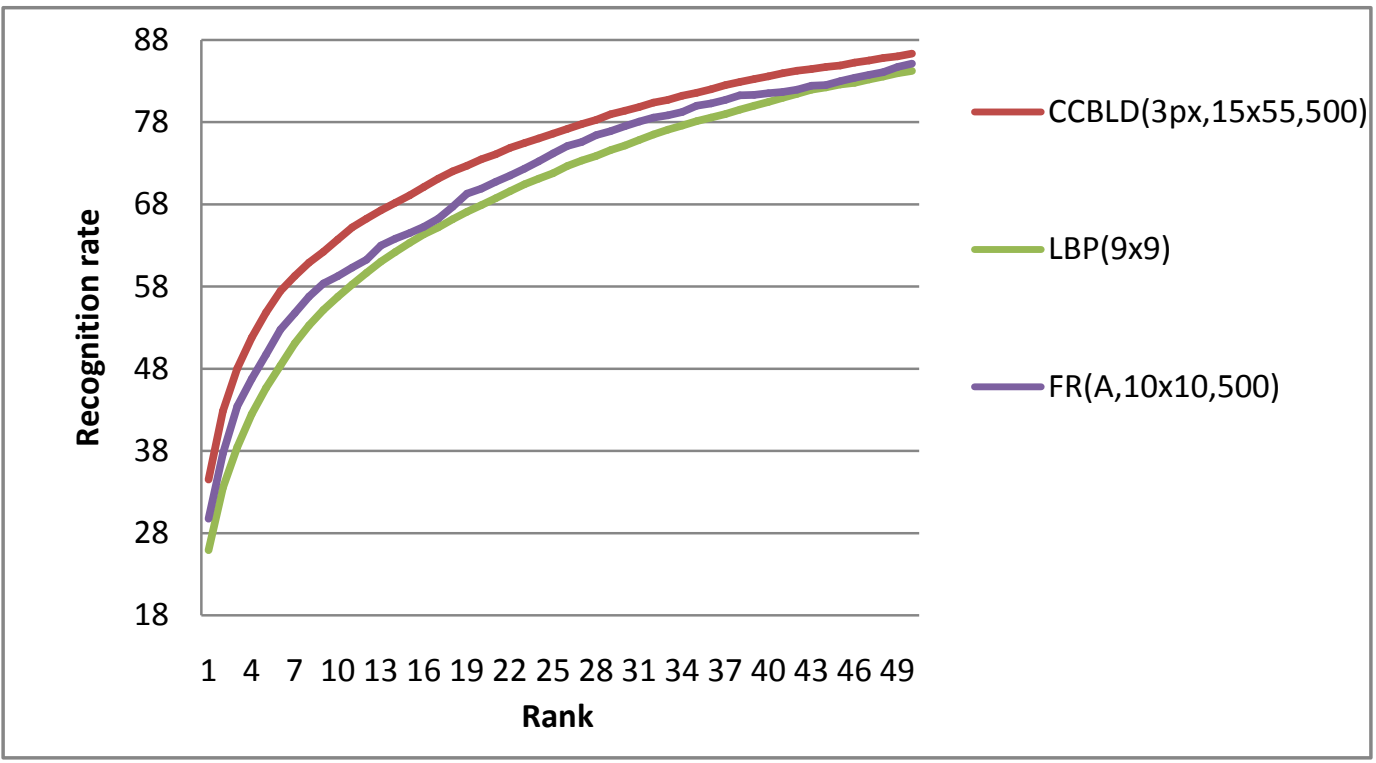

Fig. 16 A comparison of rank 1 - rank 50recognition rates (\%) for the chosen methods in group of CCBLD, LBP, and FR obtained with FG-NET images after preprocessing

\subsection{Computing time}

In this section we demonstrate the average run times for chosen operations such as the time needed to creating dictionary, changing the text files covering the words describing the pixels of the corresponding images to the text files covering the words from the dictionary, creating histogram of words describing the image, and finally the time of comparison of two such histograms. The set of images chosen to this kind of experiments was the subset of 600 grayscale images from the FERET database (see section 3.2 for details). The values illustrating the dependencies of the speed of the algorithm upon the dictionary length, block size, and division of an image are included in Table 6, Table 7, and Table 8, respectively. As 
it is seen from the tables the length of the dictionary has the most influence on the time of realization of the program.

Table 6Computing time (in ms) of selected operations for the CCBLD version with block size $1 \times 1$, no division of an image, and dictionary length changing from 100 to 1000 words

\begin{tabular}{lllllllllll}
\hline \multicolumn{1}{c}{ Dictionary length } & \multicolumn{1}{l}{$l$} \\
\hline Action & 100 & 200 & 300 & 400 & 500 & 600 & 700 & 800 & 900 & 1000 \\
\hline Creating dictionary & 561 & 2636 & 5163 & 11529 & 19923 & 29001 & 40544 & 55474 & 74823 & 94489 \\
Changing text image using dictionary & 1950 & 3532 & 5229 & 6855 & 8540 & 10445 & 12548 & 14017 & 15819 & 17714 \\
Creating histogram & 193 & 196 & 197 & 198 & 198 & 204 & 200 & 199 & 201 & 200 \\
Comparing two histograms & 0.24 & 0.36 & 0.49 & 0.62 & 0.74 & 0.86 & 0.97 & 1.09 & 1.21 & 1.32 \\
\hline
\end{tabular}

Table 7Computing time(in ms) of selected operations for the CCBLD version; no division of an image, dictionary length set to 100, and block size changing from 1x1 to 15x15

\begin{tabular}{lllllllll}
\hline \multicolumn{1}{l}{ Block size } & & & & & & \\
\hline Action & $1 \times 1$ & $3 \times 3$ & $5 \times 5$ & $7 \times 7$ & $9 \times 9$ & $11 \times 11$ & $13 \times 13$ & $15 \times 15$ \\
\hline Creating dictionary & 561 & 187 & 171 & 124 & 140 & 140 & 124 & 124 \\
Changing text image using dictionary & 1950 & 2033 & 2119 & 2075 & 2071 & 2008 & 1972 & 1982 \\
Creating histogram & 193 & 194 & 194 & 194 & 195 & 193 & 193 & 206 \\
Comparing two histograms & 0.24 & 0.24 & 0.24 & 0.25 & 0.24 & 0.24 & 0.24 & 0.24 \\
\hline
\end{tabular}

Table 8Computing time(in ms) of selected operations for the CCBLD version; no division of an image, dictionary length set to 100 , block size set to $1 \times 1$, and the division of an image changing from $1 \times 1$ to $10 \times 10$

\begin{tabular}{lllllllllll}
\hline \multicolumn{1}{l}{ Division of the image } & \multicolumn{1}{l}{} \\
\hline Action & $1 \times 1$ & $2 \times 2$ & $3 \times 3$ & $4 \times 4$ & $5 \times 5$ & $6 \times 6$ & $7 \times 7$ & $8 \times 8$ & $9 \times 9$ & $10 \times 10$ \\
\hline Creating dictionary & 561 & 452 & 670 & 561 & 702 & 468 & 655 & 483 & 530 & 670 \\
Changing text image using dictionary & 1950 & 1967 & 1901 & 1935 & 1958 & 1896 & 1912 & 1946 & 2003 & 1948 \\
Creating histogram & 193 & 193 & 195 & 196 & 198 & 200 & 203 & 204 & 208 & 211 \\
Comparing two histogram & 0.24 & 0.62 & 1.23 & 2.07 & 3.14 & 4.42 & 5.89 & 7.6 & 9.44 & 11.62 \\
\hline
\end{tabular}

\subsection{Noise/occlusion presence}

In this subsection we study the case of noise or occlusion artificially added to the images. We have modified the images using Gaussian and salt and pepper noise to the images coming from AT\&T and grayscale FERET datasets, respectively. The results are included in Table 9. Moreover, we have modified the images adding white rectangles of random size and placement which sizes were not more than 30x60 pixels in case of AT\&T and 90x150 pixels in case of FERET. We have chosen the parameters giving the best results for original images. Furthermore, in one case we changed the way of calculating the value representing a block of pixels. Namely, instead of average we have chosen the median. This simple operation makes the algorithm more robust to the noise when compared to the other methods.

Table 9Comparison of recognition rates (in percentage) for Gaussian noise, salt \& pepper noise, and occlusion

\begin{tabular}{|c|c|c|c|c|c|c|c|c|c|c|c|c|}
\hline & Noise & Gauss & n noise & & & & Salt ar & peppeı & oise & & & Occlusion \\
\hline Dataset & Method & $10 \%$ & $20 \%$ & $30 \%$ & $40 \%$ & $50 \%$ & $10 \%$ & $20 \%$ & $30 \%$ & $40 \%$ & $50 \%$ & \\
\hline \multirow[t]{3}{*}{ AT\&T } & CCBLD $(1 p x, 4 x 4,100)$ & 83.88 & 61.50 & 36.43 & 19.75 & 13.95 & 81.25 & 61.55 & 37.08 & 18.30 & 9.08 & 36.35 \\
\hline & $\begin{array}{l}\text { CCBLD(7px,3x3,400) - } \\
\text { average of pixel block }\end{array}$ & 97.43 & 95.40 & 93.58 & 90.90 & 87.48 & 97.45 & 95.60 & 95.83 & 93.70 & 89.38 & 63.30 \\
\hline & $\begin{array}{l}\text { CCBLD(7px, 3x3,400) - } \\
\text { median of pixel block }\end{array}$ & 97.23 & 96.85 & 95.93 & 95.90 & 95.28 & 97.65 & 96.83 & 96.23 & 95.85 & 95.48 & 69.30 \\
\hline
\end{tabular}




\begin{tabular}{|c|c|c|c|c|c|c|c|c|c|c|c|c|}
\hline & LBP & 83.68 & 61.20 & 33.58 & 16.08 & 6.88 & 83.53 & 62.83 & 39.50 & 21.58 & 11.40 & 32.23 \\
\hline & $\mathrm{FR}(\mathrm{D}, 5 \times 5,300)$ & 33.63 & 19.58 & 12.68 & 5.33 & 4.38 & 31.38 & 20.35 & 10.98 & 8.48 & 5.80 & 23.53 \\
\hline \multirow[t]{3}{*}{ FERET } & CCBLD(1px,7x7,400) & 67.93 & 61.75 & 54.68 & 43.78 & 20.15 & 67.38 & 60.15 & 53.13 & 44.30 & 36.58 & 7.08 \\
\hline & LBP & 63.95 & 61.18 & 53.60 & 49.68 & 33.45 & 62.30 & 56.85 & 55.18 & 53.70 & 44.83 & 7.53 \\
\hline & $\mathrm{FR}(\mathrm{D}, 4 \times 4,500)$ & 54.95 & 49.4 & 43.73 & 30.53 & 16.28 & 53.48 & 47.85 & 41.10 & 32.33 & 21.25 & 1.43 \\
\hline
\end{tabular}

\subsection{Similarity/dissimilarity measures performance}

The last statistics presented here is the assessment of performance of 4 well-known similarity/dissimilarity measures used to compare the histograms of words, namely the Hellinger, chi-square, histogram intersection, and correlation measure, see Table 10. Taking into account these results we use the Hellinger measure to compare all the histograms in our proposal. However, histogram intersection would be a good alternative here. Some introductory results in this scope were obtained in [49] for the leave-one-out technique of classification.

Table 10Comparison of recognition rates (in percentage) for Gaussian noise, salt \& pepper noise, and occlusion for AT\&T image dataset

\begin{tabular}{lllll}
\hline Method & Hellinger & Histogram intersection & Chi-square & Correlation \\
\hline CCBLD(1px,4x4,100) & 96.20 & 95.88 & 93.30 & 93.80 \\
LBP & 97.00 & 96.83 & 97.00 & 93.20 \\
FR(D,5x5,300) & 94.23 & 94.93 & 94.90 & 93.53 \\
\hline
\end{tabular}

\section{Conclusions and future work}

In the study, we have comprehensively examined the local descriptor called Chain CodeBased Local Descriptor. Moreover, we have extended its form to the description of the blocks of pixels surrounding the central block. We have discussed its application to various problems of face recognition by considering different publicly available datasets. The experimental results show that our approach is comparable or even outperforms other local descriptorsbased methods such as LBP or Full Ranking. Future studies may contain, among others, the examination of the dependence of the gallery and testing set sizes on the recognition rate, the problem of high resolution images and the optimization of the algorithm using other than Levenshtein word similarity measures, histogram of words smoothing and inventing other methods of its construction, for instance, incorporation of the color information, an application to other fields of biometrics or image analysis such as texture analysis or noise detection, examination of various methods of finding the paths constituting chain codes, or, finally, the invariance problem. In addition, it is worth to study a combination of CCBLD with deep learning.

\section{Acknowledgements}

The authors are supported by National Science Centre. Poland (grant no. 2014/13/D/ST6/03244). Support from the Canada Research Chair (CRC) program and Natural Sciences and Engineering Research Council is gratefully acknowledged (W. Pedrycz).

Part of the research in this paper use the FERET database of facial images collected under the FERET program, sponsored by the DOD Counterdrug Technology Development Program Office [54,55]. 


\section{References}

[1] T. Kanade, Computer recognition of human faces, Birkhauser, Basel, 1977.

[2] M. Turk, A. Pentland, Eigenfaces for recognition, J. Cogn. Neurosci. 3 (1991) 71-86.

[3] P.N. Belhumeur, J.P. Hespanha, D.J. Kriegman, Eigenfaces vs. Fisherfaces: recognition using class specific linear projection, IEEE Trans. Pattern. Anal. Mach. Intell. 19 (1997) 711-720.

[4] L. Wiskott, J.-M. Fellous, N. Krüger, C. von der Malsburg, Face recognition by elastic bunch graph matching, IEEE Trans. Pattern. Anal. Mach. Intell. 19 (1997) 775-779.

[5] P.J. Phillips, Support vector machines applied to face recognition, Adv. Neural Inf. Process. Syst. 11 (1998) 803-809.

[6] S. Xie, S. Shan, X. Chen, J. Chen, Fusing local patterns of Gabor magnitude and phase for face recognition. IEEE Trans. Image Process. 19 (5) (2010) 1349-1361.

[7] M. Bereta, P. Karczmarek, W. Pedrycz, M. Reformat, Local descriptors in application to the aging problem in face recognition, Pattern Recognit. 46 (2013) 2634-2646.

[8] K.-C. Kwak, W. Pedrycz, Face recognition: a study in information fusion using fuzzy integral, Pattern Recognit. Lett. 26 (2005) 719-733.

[9] P. Melin, C. Felix, O. Castillo, Face recognition using modular neural networks and the fuzzy Sugeno integral for response integration, Int. J. Intell. Syst. 20 (2005) 275-291.

[10] J. Wright, A.Y. Yang, A. Ganesh, S.S. Sastry, Y. Ma, Robust face recognition via sparse representation, IEEE Trans. Pattern Anal. Mach. Intell. 31 (2) (2009) 210-227.

[11] G.B. Huang, H. Lee, E. Learned-Miller, Learning hierarchical representations for face verification with convolutional deep belief networks, in: Computer Vision and Pattern Recognition (CVPR). 2012 IEEE Conference on, 2012, pp. 2518-2525.

[12] Y. Sun, X. Wang, X. Tang, Deep learning face representation from predicting 10.000 classes, in: The IEEE Conference on Computer Vision and Pattern Recognition (CVPR), 2014, pp. 1891-1898.

[13] D. Kurach, D. Rutkowska, E. Rakus-Andersson, Face classification based on linguistic description of facial features,in: L. Rutkowski, M. Korytkowski, R. Scherer,R. Tadeusiewicz, L.A. Zadeh,J.M. Zurada (Eds.),ICAISC 2014. Part II. LNAI 8468, 2014, pp. 155-166.

[14] P. Karczmarek, A. Kiersztyn, P. Rutka, W. Pedrycz, Linguistic descriptors in face recognition: A literature survey and the perspectives of future development, in: Signal Processing: Algorithms, Architectures, Arrangements, and Applications (SPA), 2015, Poznan. 2015, pp. 98-103.

[15] T. Ahonen, A. Hadid, M. Pietikäinen, Face recognition with local binary patterns, in: Proceedings of the 8th European Conference on Computer Vision, LNCS 3021, 2004, pp. 469-481.

[16] M. Heikkilä, M. Pietikäinen, C. Schmid, Description of interest regions with local binary patterns, Pattern Recognit. 42 (2009) 425-436.

[17] T. Ojala, M. Pietikäinen, D. Harwood, A comparative study of texture measures with classification based on feature distributions, Pattern Recognit. 29 (1996) 51-59. 
[18] T. Ojala, M. Pietikäinen, T. Mäenpää, Multiresolution gray-scale and rotation invariant texture classification with local binary patterns, IEEE Trans. Pattern Anal. Mach. Intell. 24 (2002) 971-987.

[19] M. Heikkilä, M. Pietikäinen, C. Schmid, Description of interest regions with centersymmetric local binary patterns, in: Indian Conference on Computer Vision, Graphics and Image Processing (ICVGIP) 2006, Lecture Notes in Computer Science 4338, 2006, pp. 58-69.

[20] H. Jin, Q. Liu, H. Lu, X. Tong, Face detection using improved LBP under Bayesian framework, in: International Conference on Image and Graphics, 2004, pp. 306-309.

[21] C.-H. Chan, J. Kittler, K. Messer, Multi-scale local binary pattern histograms for face recognition, in: S.-W. Lee, S.Z. Li (Eds.), ICB 2007, Lecture Notes in Computer Science 4642, 2007, pp. 809-818.

[22] S. Liao, X. Zhu, Z. Lei, L. Zhang, S. Li, Learning multi-scale block local binary patterns for face recognition, in: Advances in Biometrics. International Conference, ICB 2007, Lecture Notes in Computer Science 4642, 2007, pp. 828-837.

[23] J. Chen, S. Shan, C. He, G. Zhao, M. Pietikäinen, X. Chen, W. Gao, WLD: a robust local image descriptor, IEEE Trans. Pattern Anal. Mach. Intell. 32 (2010) 1705-1720.

[24] W. Zhang, S. Shan, W. Gao, X. Chen, H. Zhang, Local Gabor binary pattern histogram sequence (LGBPHS): a novel non-statistical model for face representation and recognition, in: Computer Vision, ICCV 2005, Tenth IEEE International Conference on, 2005, pp. 786-791.

[25] B. Zhang, S. Shang, X. Chen, W. Gao, Histogram of Gabor phase pattern (HGPP): a novel object representation approach for face recognition. IEEE Trans. Image Process. 16 (2007) 57-68.

[26] Y. Guo, Z. Xu, Local Gabor phase difference pattern for face recognition, in: Pattern Recognition, 2008, ICPR 2008, 19th International Conference on, 2008, pp. 1-4.

[27] B. Jun, H. Lee, J. Lee, D. Kimy, Statistical face image preprocessing and nonstatistical face representation for practical face recognition, in: Signal Processing and Information Technology (ISSPIT), 2009 IEEE International Symposium on, 2009, pp. 392-397.

[28] E. Abusham, H. Bashir, Face recognition using local graph structure (LGS). HumanComputer Interaction. Interaction Techniques and Environments, Lecture Notes in Computer Science 6762, 2011, pp. 169-175.

[29] Z. Lei, T. Ahonen, M. Pietikäinen, S. Li, Local frequency descriptor for low-resolution face recognition, in: Automatic Face \& Gesture Recognition and Workshops (FG 2011), 2011 IEEE International Conference on, 2011, pp. 161-166.

[30] J. Choi, W. Schwartz, H. Guo, L. Davis, A complementary local feature descriptor for face identification, in: Applications of Computer Vision (WACV), 2012 IEEE Workshop on, 2012, pp. 121-128.

[31] Z. Chai, Z. Sun, T. Tan, H. Méndez-Vázquez, Local salient patterns - A novel local descriptor for face recognition, in: International Conference on Biometrics, 2013, pp. 1-6.

[32] M. Abdullah, S. Sayeed, K. Muthu, H. Bashier, A. Azman, S. Ibrahim, Face recognition with symmetric local graph structure (SLGS), Expert Syst. Appl. 41 (2014) 6131-6137.

[33] K.-C. Fan, T.-Y. Hung, A novel local pattern descriptor-local vector pattern in high-order derivative space for face recognition, IEEE Trans. Image Process. 23 (2014) 2877-2891. 
[34] C.-Y. Su, J.-F. Yang, Histogram of gradient phases: a new local descriptor for face recognition, IET Comput. Vis. 8 (2014) 556-567.

[35] D. Gragnaniello, G. Poggi, C. Sansone, L. Verdoliva, Local contrast phase descriptor for fingerprint liveness detection, Pattern Recognit. 48 (2015) 1050-1058.

[36] S. Hegenbart, A. Uhl, A scale- and orientation-adaptive extension of local binary patterns for texture classification, Pattern Recognit. 48 (2015) 2633-2644.

[37] Z. Li, D. Gong, X. Li, D. Tao, Learning compact feature descriptor and adaptive matching framework for face recognition, IEEE Trans. Image Process. 24 (2015) 2736-2745.

[38] A. Maqueda, C.R. del-Blanco, F. Jaureguizar, N. García, Human-computer interaction based on visual hand-gesture recognition using volumetric spatiograms of local binary patterns, Comput. Vis. Image Und. 141 (2015) 126-137.

[39] J. Ren, X. Jiang, J. Yuan, Learning LBP structure by maximizing the conditional mutual information, Pattern Recognit. 48 (2015) 3180-3190.

[40] M. Smiatacz. J. Rumiński, Local texture pattern selection for efficient face recognition and tracking, in: R. Burduk, K. Jackowski, M. Kurzyński, M. Woźniak, A. Żołnierek (Eds.), Proceedings of the 9th International Conference on Computer Recognition Systems CORES 2015, pp. 359-368.

[41] Y.-T. Luo, L.-Y. Zhao, B. Zhang, W. Jia, F. Xue. J.-T. Lu, Y.-H. Zhu, B.-Q. Xu, Local line directional pattern for palmprint recognition, Pattern Recognit. 50 (2016) 26-44.

[42] J. Ylioinas, N. Poh, J. Holappa, M. Pietikäinen, Data-driven techniques for smoothing histograms of local binary patterns, Pattern Recognit. 60 (2016) 734-747.

[43] S. Bu, P. Han, Z. Liu, J. Han, H. Lin, Local deep feature learning framework for 3D shape, Comput. Graph. 46 (2015) 117-129.

[44] A. Kumar, R. Ranjan, V. Patel, R. Chellappa, Face alignment by local deep descriptor regression, 2016, arXiv:1601.07950v1

[45] Z. Ren, Y. Deng, Q. Dai, Local visual feature fusion via maximum margin multimodal deep neural network, Neurocomputing 175 (2016) 427-432.

[46] M. Bereta, W. Pedrycz, M. Reformat, Local descriptors and similarity measures for frontal face recognition: A comparative analysis, J. Vis. Commun. Image. R. 24 (2013) 1213-1231.

[47] C.H. Chan, F. Yan, J. Kittler, K. Mikolajczyk, Full ranking as local descriptor for visual recognition: A comparison of distance metrics on $s_{n}$, Pattern Recognit. 48 (2015) 13281336.

[48] J. Sivic, A. Zisserman, Video Google: A text retrieval approach to object matching in videos, in: Proceedings, Ninth IEEE International Conference on Computer vision, vol. 2, 2003, pp. 1470-1477.

[49] P. Karczmarek, A. Kiersztyn, W. Pedrycz, P. Rutka, Chain code-based local descriptor for face recognition, in: R. Burduk, K. Jackowski, M. Kurzyński, M. Woźniak, A. Żołnierek (Eds.), Proceedings of the 9th International Conference on Computer Recognition Systems CORES 2015, pp. 307-316.

[50] V.I. Levenshtein, Binary codes with correction for deletions and insertions of the symbol 1, Probl. Peredachi Inf. 1 (1965) 12-25.

[51] G. Navarro, A guided tour to approximate string matching, ACM Comput. Surv. 33 (2001) 31-88. 
[52] OpenCV 2.4.13.0 documentation, http://docs.opencv.org/2.4/doc/tutorials/imgproc/histograms/histogram_comparison/histog ram_comparison.html, 2016 (accessed 16.07.22).

[53] AT\&T Laboratories Cambridge, The Database of Faces,http://www.cl.cam.ac.uk/research/dtg/attarchive/facedatabase.html, 2016 (accessed 16.05.12).

[54] P.J. Phillips, J. Wechsler,J. Huang, P. Rauss, The FERET database and evaluation procedure for face recognition algorithms, Image Vis. Comput. 16 (1998) 295-306.

[55] P.J. Phillips, H. Moon, S.A. Rizvi, P.J. Rauss, The FERET evaluation methodology for face recognition algorithms, IEEE Trans. Pattern Anal.Mach. Intell.22 (2000)1090-1104.

[56] W. Gao, B. Cao, S. Shan, X. Chen, D. Zhou, X. Zhang, D. Zhao, The CAS-PEAL largescale Chinese face database and baseline evaluations, IEEE Trans. System Man Cybern. A 38 (2008) 149-161.

[57] Essex Collection of Facial Images,http://cswww.essex.ac.uk/mv/allfaces/, 2006 (accessed 16.05.24).

[58] A. Lanitis, Comparative evaluation of automatic age-progression methodologies, EURASIP J. Adv. Signal Process. 2008 (2008), doi: 10.1155/2008/239480. 